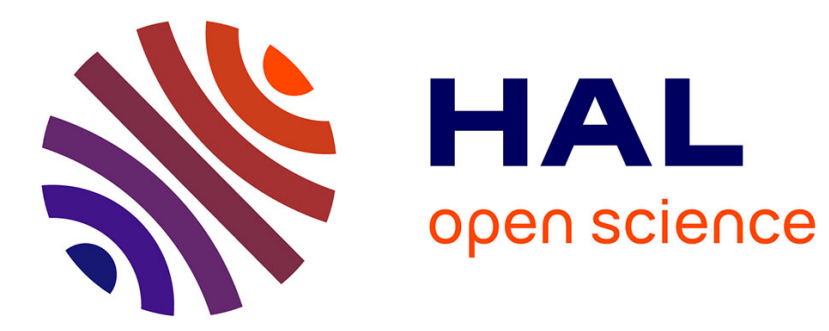

\title{
Applications of Infrared Absorption Spectroscopy to the Microelectronic Industry
}

\author{
Y. Chabal, M. Weldon, V. Marsico
}

\section{To cite this version:}

Y. Chabal, M. Weldon, V. Marsico. Applications of Infrared Absorption Spectroscopy to the Microelectronic Industry. Journal de Physique IV Proceedings, 1997, 07 (C6), pp.C6-3-C6-17. 10.1051/jp4:1997601 . jpa-00255700

\section{HAL Id: jpa-00255700 https://hal.science/jpa-00255700}

Submitted on 1 Jan 1997

HAL is a multi-disciplinary open access archive for the deposit and dissemination of scientific research documents, whether they are published or not. The documents may come from teaching and research institutions in France or abroad, or from public or private research centers.
L'archive ouverte pluridisciplinaire HAL, est destinée au dépôt et à la diffusion de documents scientifiques de niveau recherche, publiés ou non, émanant des établissements d'enseignement et de recherche français ou étrangers, des laboratoires publics ou privés. 


\title{
Applications of Infrared Absorption Spectroscopy to the Microelectronic Industry
}

\author{
Y.J. Chabal, M.K. Weldon and V.E. Marsico \\ Bell Laboratories, Lucent Technologies, Murray Hill,. NJ 07974, U.S.A.
}

\begin{abstract}
Silicon oxide and hydrogen are ubiquitous in materiais and processing issues in microelectronics. This paper reviews the value of infrared absorption spectroscopy to characterize the chemical and structural nature of silicon oxides, including buried oxides, and the presence of hydrogen both at silicon surfaces and in silicon oxides. Results involving the wet chemical cleaning of silicon and the fabrication issues of Silicon-on-Insulator are presented. Particular emphasis is given to the characterization of buried interfaces, for which IR spectroscopy is particularly useful.
\end{abstract}

L'oxyde de silicium et l'hydrogène jouent un role important dans la fabrication de matériaux pour les composants microéléctroniques. Cet article présente la spectroscopie infrarouge comme une technique de grande valeur pour caractériser chimiquement et structurellement l'oxyde de silicium, tant en surface qu'enfoui, et pour detecter la présence d'hydrogène aux surfaces et dans l'oxyde de silicium. Les résultats présentés comprennent le décapage en solutions chimiques et les problèmes de fabrication de matériau Silicium-sur-Isolant. Cette présentation est centrée en particulier sur la caractérisation des interfaces internes, pour lesquelles la spectroscopie infrarouge est particulièrement utile.

\section{INTRODUCTION}

The microelectronic industry is facing new challenges that require a more fundamental understanding of both surface and interface phenomena. The drive for ultra-small ULSI has uncovered areas where the quality of the interfaces can limit performance and yield. For example, the nature of wet chemically cleaned silicon surfaces plays an important role for subsequent processing steps to form ultra-thin gate oxides. The nature of buried layers such as oxides can also critically affect the performance of devices built on silicon-on-insulator (SOI) substrates. As a result, a precise characterization of interface formation and structure is necessary.

Vibrational spectroscopy has already played a central role in identifying the chemical termination of wet-chemically etched silicon surfaces ${ }^{1}$ and is particularly well suited to the study of buried interfaces. ${ }^{2}$ In this paper, we show how the traditional infrared absorption spectroscopy, using multiple internal reflection spectroscopy (MIR), can be extended to the interesting frequency range $\left(600-1500 \mathrm{~cm}^{-1}\right)$ where the characteristic vibrations of atoms heavier than $H$ and $D$ (such as oxides) can be probed. We also describe the novel geometry of multiple internal transmission (MIT) that makes it possible to probe buried interfaces sensitively. ${ }^{2}$

Wet chemical cleaning of silicon represent as much as 30 percent of all processing steps. The purpose is not only to clean, but also to passivate the surfaces prior to further processing. The chemical passivation and overall structure of the surface upon wet cleaning must therefore be well controlled. While IR absorption spectroscopy has been particularly useful to identify the $\mathrm{H}$ termination of silicon surfaces etched in $\mathrm{HF}$ solutions, it is now possible to obtain detailed information on the thin (4 $\AA$ ) oxide passivation after peroxide cleans, such as the RCA or Shiraki cleans, ${ }^{3}$ as illustrated in this review.

The growth of ultra-thin gate oxides can be done either from $\mathrm{H}$-terminated (HF etched) or oxideterminated (RCA process) silicon surfaces. In both cases, the quality of the ultimate oxide and of its interfaces depends in part of the initial thermal evolution of the surface under ambient gas, such as oxygen. Infrared absorption spectroscopy is becoming a useful tool to monitor the chemical and structural evolution of both $\mathrm{H}$-terminated and oxide-terminated silicon surfaces under a variety of conditions.

With the demands for low power, high speed circuitry, a new type of substrate is gaining recognition. The silicon-on-insulator (SOI) substrate is composed of a thin $(1000 \AA$ to $1 \mu \mathrm{m})$ crystalline Si film, a buried 
oxide (1000 to $5000 \AA$ A thick), and a thick $(0.5 \mathrm{~mm})$ crystalline Si handle wafer. ${ }^{4}$ Two techniques for SOI fabrication are based on direct silicon wafer bonding, and differ only in the way a thin Si layer is obtained. Silicon wafer bonding ${ }^{5}$ is performed by bringing two clean, particle free wafers into contact and annealing the joined pair to permanently (chemically) bond the two surfaces. In the more conventional bonding/etch back method, the device wafer is subsequently ground and etched by combination of mechanical and chemical thinning. In the second newer approach, the device wafer is pre-implanted with hydrogen at a depth controlled by the energy of implantation. ${ }^{6}$ During the subsequent bonding process, the implanted region shears, leaving a thin layer of crystalline silicon attached to the bonded support wafer.

The fundamental mechanisms underlying both the wafer bonding and shearing processes are not well understood, primarily due to a lack of suitable probes of the microscopic processes occurring in these buried layers. Indeed, the depth of the buried interface $(0.5 \mathrm{~mm})$ precludes the use of most particle or high energy photon probes, while the weak van der Waals forces holding the joined wafers make it impossible to use more destructive techniques such as TEM and SIMS. Infrared absorption spectroscopy stands as a unique probe that is sensitive to species trapped at the interfaces $\left(\mathrm{H}, \mathrm{H}_{2} \mathrm{O}, \mathrm{OH}, \mathrm{NH}_{x}, \mathrm{CH}_{x}, \ldots\right)$ and can also detect the formation and evolution of oxides. The spectroscopy can characterize the local structure of implanted hydrogen and the subsequent hydrogen evolution as cracks are formed and propagate.

\section{EXPERIMENTAL}

The sensitivity of infrared spectroscopy comes from maximizing the electric field of the probing radiation at the layer under study as well as the number of passes whenever possible. Fig. 1 schematically represents transmission and reflection at a semiconductor interface, either an external surface or a buried interface. To model such system, one typically uses a macroscopic approach ${ }^{7}$ where the layer under study is characterized

by a complex dielectric function, $\tilde{\varepsilon}\left(=\mathbf{n}_{\text {layer }}{ }^{2}\right)$, and a thickness, d. Fig. 1 shows the elementary geometries with the two orthogonal polarizations used: s-polarization where the electric field is parallel to the surface or interface and p-polarization where the field is parallel to the plane of incidence, with components both parallel and normal to the interface.

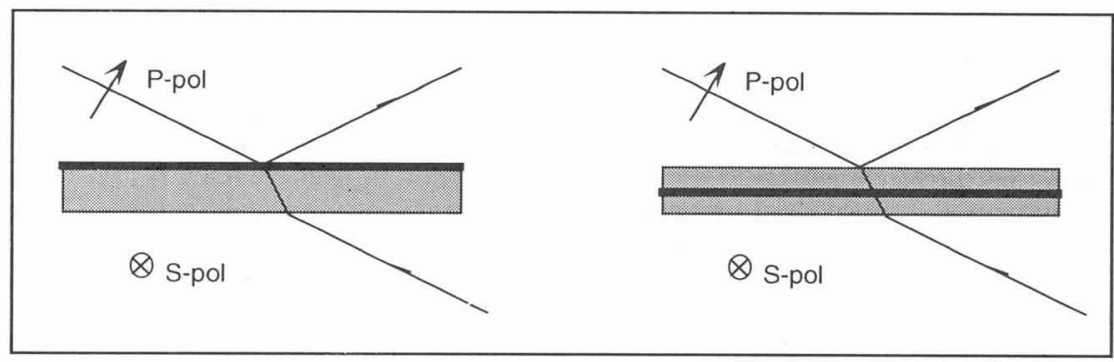

Figure 1. Schematic generic geometries for transmission, reflection and internal transmission, where the layer under study is shown as a thick line. P- and s-polarizations are also indicated by the direction of the electric field vector.

Importantly, the thicknesses under consideration range between $1 \AA$ and $100 \AA$, that is much less than the optical wavelength, $\lambda /\left|\mathrm{n}_{\text {layer }}\right|$, ranging between 1 and $20 \mu \mathrm{m}$. This implies that we can linearize (in d) all the complex absorption expressions ${ }^{8}$ and that the radiation incident on a buried interface (Fig. 1b) is essentially completely transmitted, even when the incident angle is well beyond the critical angle. Another important point is the sharp contrast between the high $\mathrm{Si}$ index $(\mathrm{n}=3.47$ at the relevant wavelengths) and the typical index of the layers under study $(\mathrm{Si}-\mathrm{H}$ with $\mathrm{n}=1.4, \mathrm{Si}-\mathrm{O}$ with $\mathrm{n}=1.5, \ldots)$. The relatively large value of the ratio $\mathrm{n}_{\mathrm{S}} / \mathrm{n}_{\text {layer }}$ l plays an important part in the sensitivity factors summarized below.

To calculate the sensitivities, the expressions $1-I / I_{0}$ and $1-R / R_{0}$ are evaluated for transmission and reflection respectively, where $I_{0}$ and $R_{0}$ are the transmission and reflection in the absence of the layer under 
study $(d=0)$. Experimentally, the surfaces chosen for reference (i.e. to measure $I_{0}$ and $R_{0}$ ) are the $H$-terminated surfaces to study an oxides, and oxidized surfaces to study H-termination, or a clean surface (no adsorbate) to study any adsorbate in a UHV environment. The key result of such calculations is that, in the linear approximation $(\mathrm{d} \ll \lambda)$, the absorption expressions for p-polarization have two terms linear in $\mathrm{d}:{ }^{27}$ one proportional to the imaginary part of $\tilde{\varepsilon}, \operatorname{Im}(\tilde{\varepsilon} \mathrm{d})$, an the other proportional to the imaginary part of $1 / \widetilde{\varepsilon}, \operatorname{Im}(\mathrm{d} / \tilde{\varepsilon})$ :

$$
1-\mathrm{I} / \mathrm{I}_{0}=\frac{2 \pi \mathrm{d}}{\lambda} \frac{1}{\mathrm{n} \cos \theta}\left[\mathrm{S}_{\mathrm{S}} \operatorname{Im}(\tilde{\varepsilon} \mathrm{d})+\mathrm{S}_{\mathrm{p}} \operatorname{Im}\left(\frac{1}{\tilde{\varepsilon}} \mathrm{d}\right)\right]
$$

while the expression for s-polarization has only one term proportional to $\operatorname{Im}(\tilde{\varepsilon} \mathrm{d})$ :

$$
1-\mathrm{I} / \mathrm{I}_{0}=\frac{2 \pi \mathrm{d}}{\lambda} \frac{1}{\mathrm{n} \cos \theta}\left[\mathrm{S}_{\mathrm{S}}^{\mathrm{S}-\mathrm{pol}} \operatorname{Im}(\tilde{\varepsilon} \mathrm{d})\right] .
$$

The index of refraction is that of the substrate(e.g. $n=n_{S i}$ ) and $\theta^{\prime}$ is the incidence angle on the layer under study. The quantity $S_{s}, S_{p}$ and $S_{s}^{s-p o l}$ are proportionality factors that involve the angle of incidence and all the indices of refraction and can be viewed as sensitivity factors that differentiate specific geometries. Each term in Eqs. (1) and (2) has a simple physical origin: the first corresponds to the absorption of the vibrational components parallel to the surface or interface as can be seen for the expression of s-polarization in Eq. 2; this term is typical of optical absorption in the bulk where the electric field is always normal to the propagation vector. The second term is proportional to the absorption of the vibrational components normal to the surface or interface, and is a direct consequence of the presence of an optical interface and non-normal incidence. This second term involves a component of the electric field parallel to the propagation vector which corresponds to the case of the interaction of an electron field with a solid in electron energy loss spectroscopy. This second term is therefore sensitive to the longitudinal instead of the transverse modes of elementary vibrations. The use of p-polarization is therefore best to study both the TO and LO absorptions of thin films. We note in passing that spectroscopy of metal surfaces is limited to the LO modes due to the strong cancellation of all parallel components of the electric field (selection rules).

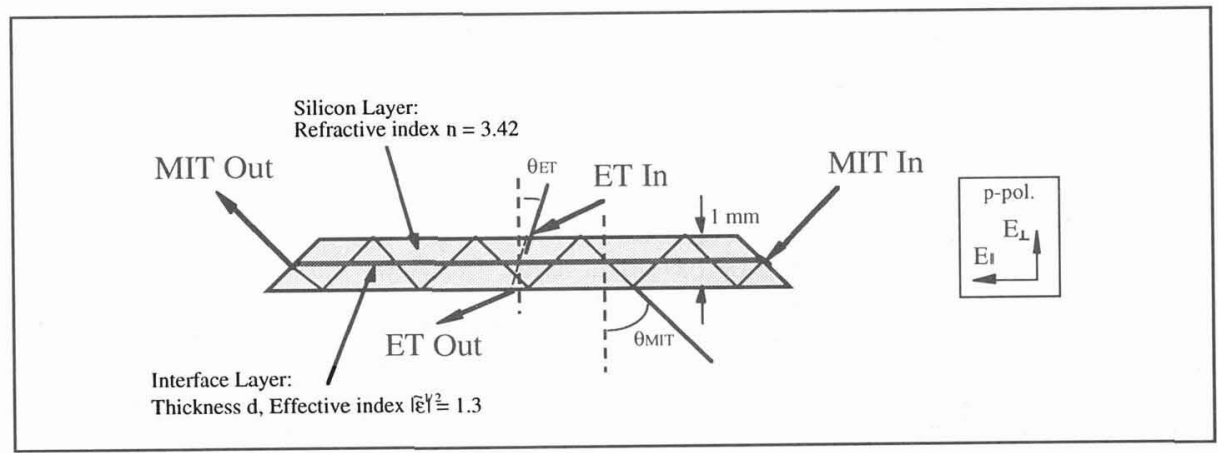

Figure 2. Illustration of the geometries used either for Multiple Internal Transmission (MIT) when the layer is sandwiched at the center, or Multiple Internal Reflection (MIR) when the layer is on the outer surfaces. Also indicated is the simple External Transmission (ET) used to study either a layer on the outer surfaces or sandwiched at the center of the plate. 
We are now in a position to consider the sensitivity of specific geometries that are the most widely used experimentally, schematically shown in Fig. 2. We define external reflection and transmission when the radiation is incident from the outside (air or vacuum side of the surface), and internal reflection and transmission when the radiation is incident from the substrate (silicon). For external surfaces, the radiation is totally internally reflected for internal incident angles greater than $\theta=17^{\circ}$ (Si). The use of external reflection is particularly inappropriate since a good fraction of the radiation is lost through refraction and the magnitude of the $1-R / R_{0}$ has a ștrong dependence (even changes sign) on the angle of incidence. For internal transmission,

on the other hand, the key result is that the sensitivity factor $S_{p}$ is proportional to $\sin ^{2}(\theta) \times n^{4} /|\tilde{\varepsilon}|^{2}$, which is in general large since $n>|\tilde{\varepsilon}|^{1 / 2}$.

For practical geometries (used before), the sensitivity factors are: ${ }^{27}$

$\mathrm{S}_{\mathrm{p}} / \mathrm{n} \cos \theta=3.3$ and $\mathrm{S}_{\mathrm{s}} / \mathrm{n} \cos \theta=0.3$ for external transmission at the Brewster angle $\left(\theta=74^{\circ}\right)$

(for both an external layer or a layer sandwiched internally);

$\mathrm{S}_{\mathrm{p}} / \mathrm{n} \cos \theta=1$ and $\mathrm{S}_{\mathrm{s}} / \mathrm{n} \cos \theta=0.8$ for internal reflection at $45^{\circ} ;$

$\mathrm{S}_{\mathrm{p}} / \mathrm{n} \cos \theta=28$ and $\mathrm{S}_{\mathrm{S}} / \mathrm{n} \cos \theta=0.2$ for internal transmission at $45^{\circ}$.

These results immediately suggest that external surfaces can be best studied using internal reflection only if the number of reflections is well over 3 and that a buried interface is always better studied using internal transmission. Moreover, the relative enhancement of the perpendicular component over the parallel component absorptions in MIR (which increases with increasing incident angle) provides a powerful way to separate the interface absorption from absorption in the bulk or at the external surfaces. This enhancement also makes it less critical to have perfectly clean outer surfaces or stable surfaces, since their contribution is so much weaker. We emphasize again that the relative enhancement comes from the large Si index compared to any layer under study.

To decide on the best geometries, we must also consider the pathlength through the $\mathrm{Si}$ in the range $\left(<1500 \mathrm{~cm}^{-1}\right)$ for which Si absorption is relevant. In the region between 1000 to $1300 \mathrm{~cm}^{-1}$ for instance, important for the $\mathrm{SiO}_{2}$ studies, the absorption coefficient varies between 1 and $5 \mathrm{~cm}^{-1}$, and is larger below $1000 \mathrm{~cm}^{-1}$. For typical wafer thicknesses $(0.05 \mathrm{~cm})$, such absorption restricts the use of multiple internal reflection (or transmission) to $1 \mathrm{~cm}$ long samples, that is to 20 reflection or passes at most. The temperature dependence of such lattice absorption, however, is the most severe experimental challenge and often limits the ability to detect weak absorptions.

\section{WET CHEMICAL CLEANING}

Wet etching has been a critical step in Si processing. Peroxides cleans are used for eliminating metal and hydrocarbon contamination and are known to form a passivating oxide layer. ${ }^{9} \mathrm{HF}$ cleans are used to get rid of silicon oxides as well as of other metallic impurities and had been known for years to leave the surface very inert. ${ }^{10}$ Initially, it was thought that fluorine-termination of the surface upon HF etching was responsible for the passivation, partly because F had been detected with XPS and AES that have no sensitivity to $\mathrm{H}$ and partly because $\mathrm{F}$ termination is a necessary step of oxide removal. In 1986, Yablonovitch et al. ${ }^{11}$ first used IR absorption spectroscopy to show that a monolayer of hydrogen was left upon HF etching. That work, along with EELS measurements, ${ }^{12}$ has motivated a series of detailed MIR studies best summarized in Fig. 3 . The two widely different spectra of Fig. 3 involving Si-H stretching modes are obtained after etching with HF solutions of two different $\mathrm{pH}(1$ and 7.8). While the complex spectrum indicates the presence of many types of hydrides $\left(\mathrm{Si}-\mathrm{H}, \mathrm{Si}-\mathrm{H}_{2}, \mathrm{Si}-\mathrm{H}_{3} \text { and sterically constrained } \mathrm{SiH}_{2}\right)^{13}$, the sharp spectrum is only detected with p-polarization and is indicative of an ideally monohydride-terminated, unreconstructed Si(111) surface. ${ }^{14}$ The interpretation is that low $\mathrm{pH}$ etching leads to atomically rough surfaces, while higher $\mathrm{pH}$ etching leads to atomically smooth surfaces, as later confirmed with STM studies. ${ }^{15}$ 'The presence of higher hydrides terminating an atomically rough surface is schematically illustrated in Fig. 4. 
Figure 3. Spectra of (a) atomically rough and (b) atomically smooth $\mathrm{H} / \mathrm{Si}(111)$ surfaces. Note the scale change for the (a).
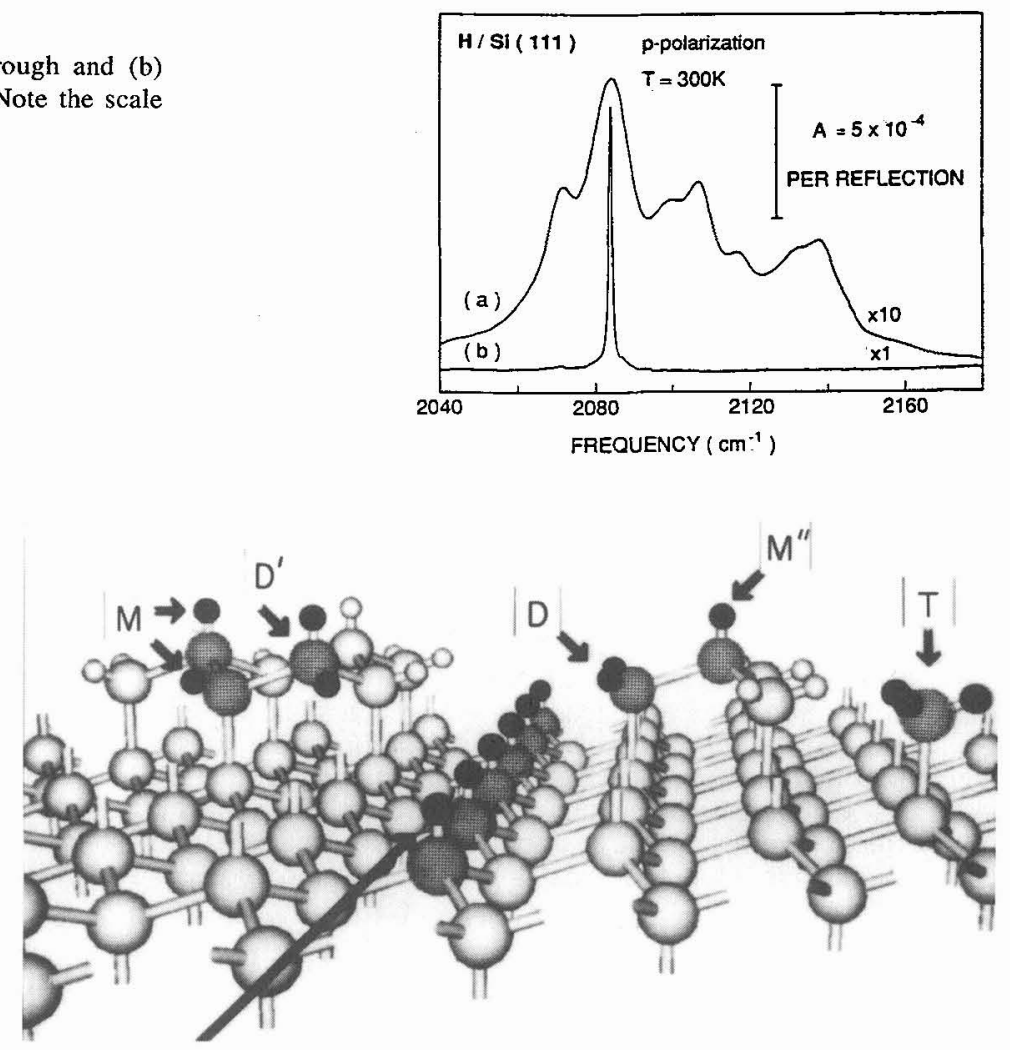

Figure 4. Computer generated Si(11) surface showing the ideal termination and other selective termination of an atomically rough surface.

The IR studies were key to establish the $H$ termination, later explained theoretically. ${ }^{16}$ The spectral analysis and polarization studies performed in air right after wet etching was critical to confirm the occurrence of preferential etching of $\mathrm{Si}$ taking place at higher $\mathrm{pH}$, subsequent to oxide removal. These studies were relatively easy because the modes of interest were the silicon stretching vibrations around $2100 \mathrm{~cm}^{-1}$ that can be studied using MIR with long samples (with typically 75 reflections). Moreover, the silicon hydrogen spectra are relatively sharp in an area of complete transparency of the silicon, making it possible to use simple background subtraction.

The situation is quite different to study the thin $(4 \AA)$ oxides formed by wet chemical cleaning since the silicon oxygen stretch modes are in the $1000-1300 \mathrm{~cm}^{-1}$ region. In this range, the silicon absorption is substantial $^{\dagger}$ and the oxide modes themselves are broad and featureless. The background subtraction is therefore very challenging. Yet, the nature of such oxides is becoming more important as they are the preferred passivation prior to the very thin gate oxides formation. Consequently, efforts are being made to develop methods to probe this difficult region.

Multiple internal reflection can be used in conjunction with a high index transparent material such as germanium that traps the radiation. ${ }^{18}$ When an oxidized silicon wafer is brought in close proximity, the evanescent fields probe the thin oxides and the silicon absorption is minimized since the effective thickness traversed is of order a few wavelengths $(20-40 \mu \mathrm{m})$ times the number of reflections (typically 50 to 100), that is less than a mm. Alternatively, a metallic layer can be manufactured by metallic implantation (e.g. Co+ implantation $)^{19}$ so as to provide a mirror for the probing IR radiation. This has the effect of increasing the electric field at the thin oxide layer and minimizing the pathlength through the Si (1000-5000 $\AA$ at most). Both approaches have been used successfully. Both approaches are subject to a strong selection rule: only the component of absorption perpendicular to the interface is detected preferentially. This limits the study to the longitudinal (LO) modes. 
Figure 5. Infrared absortion spectra of (i) wet chemical oxide prepared by an RCA clean with $\mathrm{HCl}$ peroxide as the last step, and (ii) $34 \AA$ thermal oxide. Both spectra are taken by a simple transmission at $74^{\circ}$ external incidence.

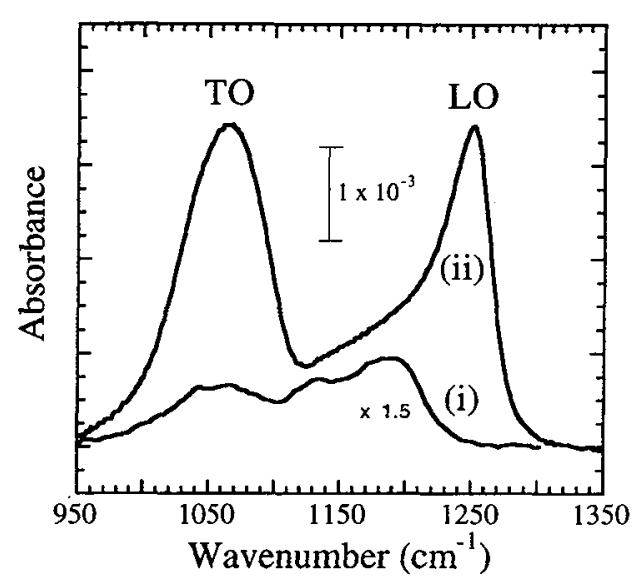

Simple transmission at the Brewster angle is however adequate to detect thin wet chemical oxides, as shown in Fig. 5(i). An advantage of this approach is the sensitivity to both the TO and LO modes. The former is sensitive to the stoichiometry, structure and strain, while the shift of the other is directly related to the Coulomb interactions that intimately depend on the matrix density and the local dynamic dipoles (polarization of the Si-O bonds). The contrast between an oxide manufactured by wet etching (Fig. 5i) and a thermal oxide (Fig. 5ii) is most evident looking at the LO modes centered at $1190 \mathrm{~cm}^{-1}$ and $1255 \mathrm{~cm}^{-1}$, respectively. The additional contribution centered at $1130 \mathrm{~cm}^{-1}$ for the chemical oxide is extremely interesting and is being investigated at present.

The interpretation of the TO and LO spectral features is complex, which accounts for the relatively modest effort made with IR absorption spectroscopy so far. Relevant factors are chemical such as bonding state directly related to stoichiometry (non-stoichiometry is defined as the departure from an average of two oxygen for each silicon atom), physical such as strain defined as the departure from perfectly tetrahedral angles or structural reorientation next to the $\mathrm{Si}$ interface, and electromagnetic such as image charge and orientation effects at the interface. Each factor has to be treated quantitatively before a consistent interpretation can be reached. This effort, requiring the development of model systems, is underway at present.

For the spectra in Fig. 5, a qualitative understanding can be reached by performing the following experiments. The wet chemical oxide can be annealed to eventually reach the stage of thermal oxide, and the thermal oxide can be etched to eventually reach the average thickness of the chemical oxide. These are difficult experiments because the conditions of annealing are extremely important (vacuum versus ambient, nature of ambient during the anneal) and the thermal oxide thinning must be uniform enough (requiring AFM monitoring at all stages, and extremely clean etching solutions). The preliminary results are shown in Fig. 6 to illustrate the possibilities rather than to reach a definite conclusion.

In conclusion, IR absorption spectroscopy is an important tool to investigate wet chemical cleaning. It is particularly powerful to study the nature of HF etched silicon, particularly in conjunction with AFM and STM. IR absorption is also adequate to study the nature of oxides, although it must be performed in conjunction with XPS and AFM measurements to be useful, considering the complexity of the quantitative analysis. In both cases, the results of IR/AFM/STM/XPS must be correlated with TXRF and electrical measurements to be fully useful for processing. In this manner, the chemical and structural nature of the surfaces can be linked to the trace impurities and eventually electrical performance of the system. 
Figure 6. Infrared absorption spectra of a thin $34 \AA$ thermal oxide as it is etched off.

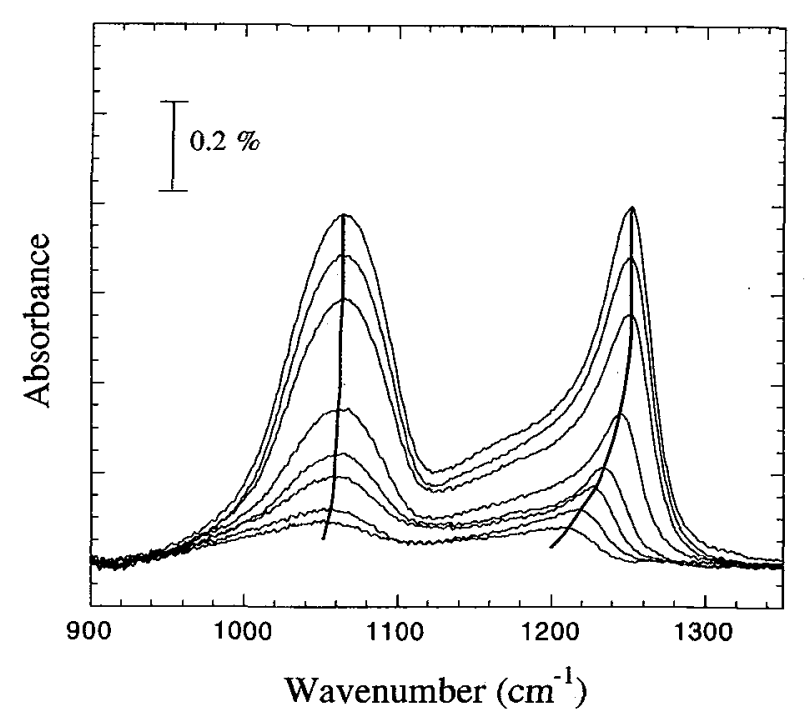

\section{SILICON-ON-INSULATOR}

Silicon-On-Insulator (SOI) describes a relatively thin crystalline silicon layer attached to an insulating layer such as $\mathrm{SiO}_{2}$ or glass. ${ }^{4}$ Generally a substrate wafer (called handle wafer) holds the above sandwich and the insulating oxide is referred to as Buried OXide (BOX). The thickness of the silicon (SOI) ranges from $500 \AA$ to tens of microns, and that of BOX from several thousand $\AA$ to several microns, although it can be the whole handle structure in the case of Si on glass. The applications for SOI wafers are growing within the microelectronic industry due to the insulating properties of such wafers: radiation hardness, low voltage (low power) or very high voltage applications, speed, high packing density and possible savings on processing steps. While the technology is used in a number of markets, the interest has risen since SOI wafers are candidate for low power, high speed CMOS circuitry, a potentially huge market. ${ }^{6}$

From a materials point of view, the challenge is to make such crystalline layers on amorphous insulators. Two main approaches have been used with surprising success. The SIMOX (Separation by IMplantation of OXygen) approach uses a high-energy oxygen implant and subsequent annealing steps to form the required silicon dioxide layer at the desired depth beneath the top silicon surface. ${ }^{20}$ The extensive annealing protocol is necessary to repair the considerable lattice disruption of the top silicon layer (later active SOI layer) caused by implantation. At present the density of threading dislocations remains at $10^{3} \mathrm{~cm}^{-2}$. The BOX and SOI thicknesses achievable by the SIMOX method are limited by the dose and energy of oxygen ions. The other approach involves direct wafer bonding to form the SOI structures and will be the subject of our studies.

With wafer bonding, a thermally oxidized wafer is joined to a handle wafer. The thickness of the oxide is chosen to match the desired BOX thickness. To obtain a thin SOI, the conventional method is to mechanically grind the device wafer (to a few microns) and then etch (eg. Plasma Assisted Chemical Etching) to leave a layer as thin as $1000 \AA$. A more novel approach is to implant the device wafer through the thick thermal oxide with high energy hydrogen ions. The wafer is then joined to the handle wafer, and the pair is annealed between 400 and $600{ }^{\circ} \mathrm{C}$, at which temperature the device wafer shears, leaving behind a thin SOI. The shearing occurs at the average $\mathrm{H}$ penetration depth, and can therefore be controlled by the energy of implantation. Infrared absorption spectroscopy has been instrumental to study the bonding and shearing mechanisms. 


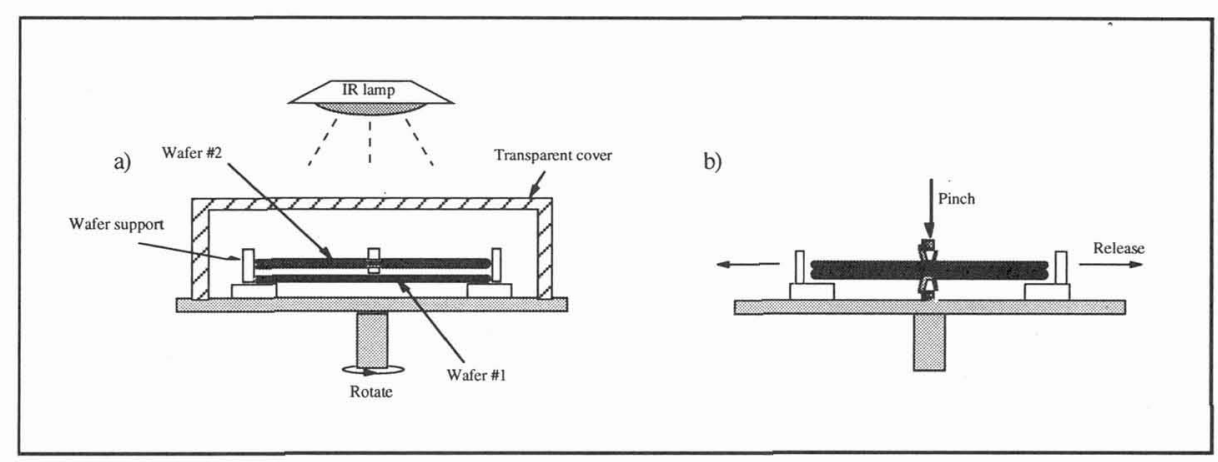

Figure 7. Schematic of the joining process

\subsection{Joining mechanism}

Joining is the phenomenon of bringing two clean, particle-free wafers and pressing at the center, as schematically shown in Fig. 7. Cleaning is done by wet chemistry leading either to H-terminated surfaces (HF etching), or oxidized surface (peroxide etching such as the RCA cleans). Particles are eliminated by DI water flushing and high speed rotation just prior to joining. The intimate physical contact initiated at the center ${ }^{21}$ propagates to the edges at a "contact wave velocity" that depends on the nature of the surface (chemical termination, flatness and roughness). IR imaging and acoustic microscopy suggest that the two internal surfaces are within $100 \AA$ in void free areas. However, there is a need to know exactly the average distance as well as to characterize the presence of impurities at the interface, prior to annealing (the final bonding step), in order to understand the nature of the forces. At room temperature, chemical bonds cannot be established so that physical forces must be operational.

\subsubsection{Hydrophobic surfaces}

We have addressed this problem by starting with a model system, the ideally $\mathrm{H}$-terminated Si(111) surface. ${ }^{14,15}$ A highly homogeneous, atomically flat surface can indeed be prepared simply in solution by etching in a solution of ammonium fluoride (high $\mathrm{pH}$ hydrofluoride solution) for a prescribed time. The resulting surface is characterized by a sharp $\left(<1 \mathrm{~cm}^{-1}\right) \mathrm{Si}-\mathrm{H}$ stretch mode at $2083.7 \mathrm{~cm}^{-1}$, polarized perpendicular to the surface (Fig. 8a). The inhomogeneous broadening is typically of order $0.05 \mathrm{~cm}^{-1}$, consistent with large atomically flat domains ( $>1000 \AA$ ), separated by atomically straight double layer steps. The chemical purity and inertness of such surfaces is the highest of any surface so that the dominant forces are likely to be van der Waals in nature.

Joining two $\mathrm{H} / \mathrm{Si}(111)-(1 \times 1)$ surfaces dramatically changes the IR absorption spectrum (Fig. 8b). For the same number of passes (MIT geometry) as reflections (MIR geometry), the absorption is substantially larger. The measured increase in integrated intensity is 25 which is in good agreement with the $S_{p}$ factors listed in section 2 (28) and beautifully confirms the enhancement derived with MIT. The appearance of a distinct band centered at $2065 \mathrm{~cm}^{-1}$ is more intriguing as it is a direct reflection of the interaction between the two surfaces. To understand its origin, we first establish that the bimodal distribution and broadening between the bands at 2065 and $2083 \mathrm{~cm}^{-1}$ is due to varying degrees of interaction between the surfaces. Fig. 8(c) shows the result of probing a smaller piece ( $8 \mathrm{~mm}$ instead of $37 \mathrm{~mm}$ in spectrum b) for which the spectrum is most perturbed from the $2083 \mathrm{~cm}^{-1}$ band. In contrast, other parts of the large sample resemble most the unperturbed surfaces. Interestingly, a further decrease in the Si-H frequency of $8 \mathrm{~cm}^{-1}$ was observed on annealing the piece shown in spectrum (c) to $700^{\circ} \mathrm{C}$ (Fig. 8d), at which temperature permanent Si-Si bonding starts. These observations suggest that the spectral broadening is arising from varying degrees of interaction (distances) between the two internal surfaces. The $2057 \mathrm{~cm}^{-1}$ band reflects the maximum interaction obtainable. The issue is whether this shift is due to trapped impurities or to a direct physical interaction between the two $\mathrm{Si}-\mathrm{H}$ surfaces.

Interface impurities could either be chemisorbed or simply physisorbed. The possibility that surface chemisorbed contaminants cause the observed shift is eliminated because the spectral shifts are reversible 
Figure 8. Demonstration of the changes that take place on joining $\mathrm{H}$-terminated $\mathrm{Si}(111)$ wafers: (a) MIR spectrum of a single $\mathrm{H} / \mathrm{Si}(111)-(1 \times 1)$ sample, (b) MIT spectrum of two H/Si(111)-(1x1) wafers joined at room temperature probed with the same number of passes as in (a), (c) MIT spectrum of a piece of joined sample using a plate that is 4.6 times shorter than in (b), and (d) MIT spectrum of sample shown in (c) after annealing to $700^{\circ} \mathrm{C}$.

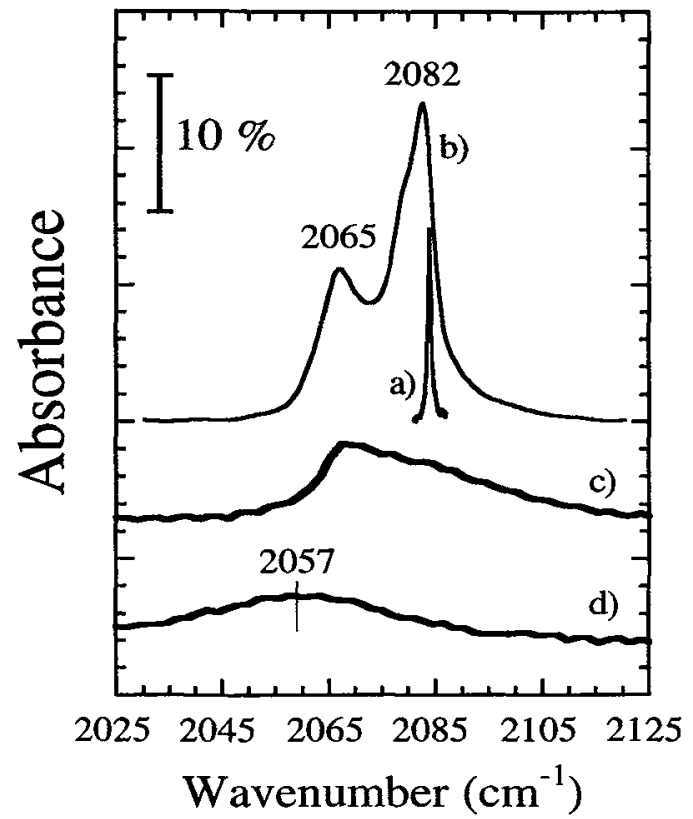

with respect to separation: the spectrum sharpens up again into a single line at $2083.5 \mathrm{~cm}^{-1}$ when the wafers are separated and broadens with a band at $2065 \mathrm{~cm}-1$ when the wafers are re-joined. The possibility that a physisorbed layer accounts for the spectral shift was checked by adsorbing a variety of atomic and molecular species at low temperatures such as $\mathrm{CO}, \mathrm{N}_{2}, \mathrm{Ne}, \mathrm{Ar}, \mathrm{Kr}$ or $\mathrm{Xe}$ and observing for each case an increase of the $\mathrm{Si}-\mathrm{H}$ stretch frequency $\left(5\right.$ to $20 \mathrm{~cm}-1$ ), the magnitude depending on the adsorbate. Trapping $\mathrm{H}_{2} \mathrm{O}$, $\mathrm{HF}$ and $\mathrm{C}_{2} \mathrm{H}_{5} \mathrm{OH}$ between the two surfaces prior to joining also led to frequency increases and increased broadening. ${ }^{7,22}$ We conclude therefore that the red shift originates from the direct interaction between the two $\mathrm{Si}-\mathrm{H}$ surfaces.

Direct interaction was investigated from first-principles calculation by D. Hamann. ${ }^{7,23}$ Based on the local density approximation, ${ }^{24}$ utilizing separable norm-conserving pseudopotentials to represent the atoms, ${ }^{25}$ and a plane-wave basis set to expand the wave functions, supercells consisting of 10 (or 12) $\mathrm{Si}$ (111) layers plus terminating $\mathrm{H}$ layers on each surface were used to simulate the two surfaces. The three-fold and atop geometries resulted from using 10 and $12 \mathrm{Si}$ layers, respectively. These calculations revealed that in both cases, the IR active mode (asymmetric $\mathrm{Si}-\mathrm{H}$ stretch) suffered a red shift of approximately $29 \mathrm{~cm}^{-1}$, in remarkable agreement with the measured $\left(27 \mathrm{~cm}^{-1}\right)$ red shift. ${ }^{23}$

This model system clearly shows that purely physical forces are sufficient to hold the wafers together. The above results show that, upon joining at room temperature, most of the macroscopic surface is within $2 \AA$. Since it is known that H-terminated (hydrophobic) surfaces attract hydrocarbons, this situation can exist only if most of the physisorbed impurities are swept away during the joining process, a truly remarkable conclusion which has important implications for using wafer bonding to replace epitaxy.

The situation is quite different if the surfaces are atomically rough. In that case, the silicon-hydrogen stretching bands are not altered upon joining, indicating that the majority of the surface remains unperturbed. ${ }^{26}$ Instead, the forces most likely to hold the two surfaces together originate at minority sites, such as chemical impurities (e.g., Si-OH) or structural defects (e.g., steps, adatoms). 
Figure 9. Top panel: typical MIT infrared spectrum of two hydrophilic Si(111) wafers, joined at room temperature with schematic representation of the normal modes associate with each spectral feature. Bottom panel: schematic depiction of the interface region that gives rise to the above spectrum with concentrations inferred from the integrated areas corrected for the sensitivity factors.
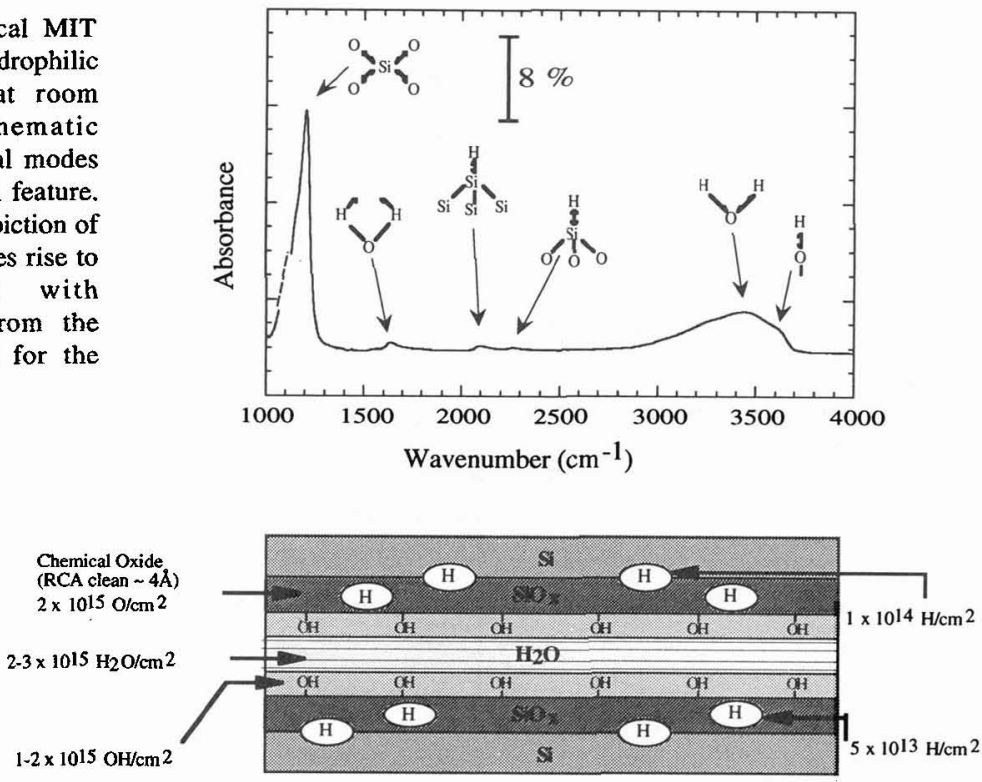

\subsubsection{Hydrophilic surfaces}

A more realistic situation is the bonding of oxidized silicon wafers, obtained by wet chemical processing described in section 3. These surfaces are terminated by hydroxyl groups that attract water molecules, hence the hydrophilic properties. Fig. 9(top) shows the spectrum obtained by MIT (short sample) of two RCA cleaned Si wafers, along with the vibrational assignments.

The first features to note are the broad band centered around $3400 \mathrm{~cm}^{-1}$, along with the weaker, sharper band at $1630 \mathrm{~cm}^{-1}$. These modes are assigned to the stretching and scissor vibrations of water molecules. The position, strength and width of the $\mathrm{O}-\mathrm{H}$ stretch are characteristic of hydrogen-bonded molecules. Although such $\mathrm{H}$-bonding is observed for physisorbed water on a single surface, the spectra are consistent with (i.e. suggest that) the primary force holding the two wafers together is the $\mathrm{H}$-bonding between the thin water layers of each internal surface. $\mathrm{H}$-bonding is stronger than van der Waals forces and in the case of atomically rough surfaces, such as oxidized Si surfaces, water is more likely to rearrange itself to make up for surface roughness, leading to a stronger "bond strength" as observed. The quantitative information obtained from the spectra is the average concentration of water, $2-3 \times 10^{15} \mathrm{H}_{2} \mathrm{O} \mathrm{cm}$, corresponding to $3-4$ monolayers. ${ }^{7.23}$

The rest of the spectrum contains contributions from $1-2 \times 10^{15}-\mathrm{OH} \mathrm{cm}^{-2}$ at $3600 \mathrm{~cm}^{-1}$, again shifted by $\mathrm{H}$-interactions with the adjacent physisorbed water molecules, $1-2 \times 10^{14} \mathrm{Si}-\mathrm{H} \mathrm{cm}^{-2}$ at $2100 \mathrm{~cm}^{-1}$, dissolved $\mathrm{H}$ in oxide at $2250 \mathrm{~cm}^{-1}$, and a total of 8 to $10 \AA$ chemical oxide characterized by the strong band at $1205 \mathrm{~cm}^{-1}$. The results are summarized schematically in Fig. 9(bottom), and the thermal evolution of these modes discussed in the next section.

In summary, two types of forces are operative to hold wafers upon room temperature joining: van der Waals and H-bonding. Van der Waals forces are most apparent (dominate) in the case of atomically flat, inert 
Figure 10. MIT spectra of two joined Si(100)-H surfaces as a function of annealing temperature; (i) room temperature (ii) after annealing to $500{ }^{\circ} \mathrm{C}$; and $600^{\circ} \mathrm{C}$. Note the presence of oxygen attached to $-\mathrm{Si}-\mathrm{H}$ groups $\left[v(\mathrm{Si}-\mathrm{H})>2150 \mathrm{~cm}^{-1}\right]$ at room temperature, which agglomerates to form predominantly $\left(\mathrm{O}_{3}\right)-\mathrm{Si}-\mathrm{H}$, on annealing above $500^{\circ} \mathrm{C}$.

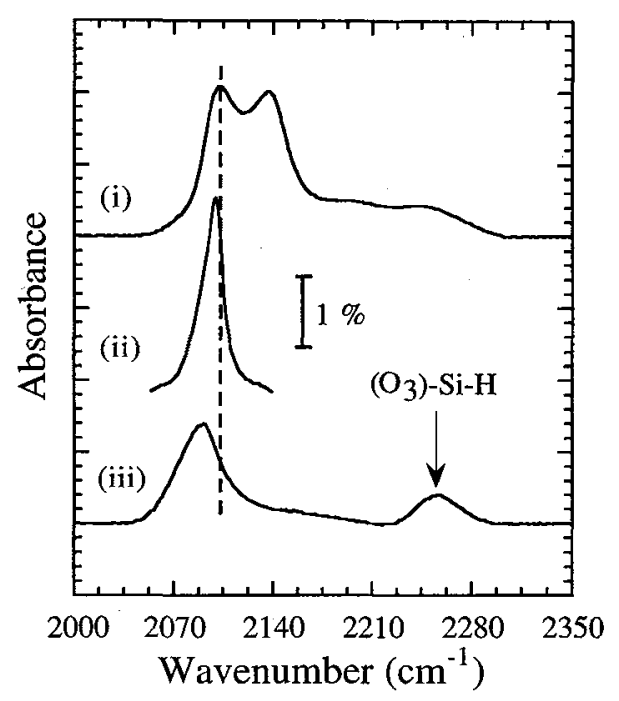

$\mathrm{H}$-terminated $\mathrm{Si}(111)$ surfaces as evidenced by a substantial $\mathrm{Si}-\mathrm{H}$ stretch shift; $\mathrm{H}$-bonding dominates in the case of $\mathrm{H}_{2} \mathrm{O}$-covered oxidized (hydrophilic) surfaces, as evidenced by a red-shifted, broad O-H stretch band. For atomically rough, hydrophobic surfaces, the situation is less clear because the majority of the inner surfaces is not affected (no spectral shift of Si-H bands), and we can only infer that a low concentration of sites is responsible for the macroscopic forces holding the two wafers together. Yet, the macroscopic bonding strength is not lower than that of atomically flat $\mathrm{H} / \mathrm{Si}(111)$, suggesting that chemical defects subject to H-bonding may be operative.

\subsection{Bonding mechanism}

Chemical bonds between the two surfaces can only be formed by high temperature annealing. IR absorption spectroscopy can be used to monitor when certain species are dissociated and, in some case, which new bonds are formed. For hydrophobic surfaces, the disappearance of $\mathrm{Si}-\mathrm{H}$ stretching vibrations is most readily monitored. In contrast to free surfaces in vacuum, the $\mathrm{Si}-\mathrm{H}$ stretching vibrations are observed well beyond $500^{\circ} \mathrm{C}$ anneal temperature (see Fig. 8d for instance). The interpretation of this observation is not that the $\mathrm{Si}-\mathrm{H}$ bonds are not broken at $500^{\circ} \mathrm{C}$, but rather that the hydrogen is rebonded and remains attached to Si upon cooling (all data are taken at room temperature), because it is trapped at the interface. At high temperature, the effective pressure at the interface is very high so that the internal surfaces remain passivated in a dynamic way on a time-average basis.

The dynamic aspect is best illustrated for atomically rough $\mathrm{H}$-terminated surfaces, for which the internal surfaces rearrange and reconstruct at high temperatures $\left(500-700^{\circ} \mathrm{C}\right)$ as shown in Fig. 10. The $\mathrm{Si}(100)$ surfaces, for instance, exhibit a dimerized monohydride reconstruction upon annealing, that is only observed upon $\mathrm{H}$ exposure on a clean $\mathrm{Si}(100)$ under UHV conditions. While the termination upon $\mathrm{HF}$ etching involves mono-, di- and tri-hydrides of an unreconstructed (although rough) surfaces (Fig. 10a), the spectrum is dominated by a single narrower band at $1098 \mathrm{~cm}^{-1}$ upon annealing that is polarized perpendicular to the interface and corresponds the symmetric stretch of the reconstructed (dimerized) monohydride observed in UHV studies (Fig. 10b). Interestingly, this mode is redshifted and its intensity decreased upon further annealing, as $\mathrm{Si}-\mathrm{Si}$ presumably form. This red shift has a similar origin to the red shift ( 2065 to $2057 \mathrm{~cm}^{-1}$ ) observed upon annealing the H/Si(111)-(1x1) joined wafers (see Fig. 8). In both cases, it is reasonable to assume that the loss of $\mathrm{Si}-\mathrm{H}$ intensity and red shift are correlated with the formation of $\mathrm{Si}$-Si bonds between the two internal surfaces, i.e. the formation of chemical bonds, consistent with a dramatic increase in bonding strength. 
The annealing process of joined hydrophilic surfaces is summarized in Fig. 11, where selected spectra are shown for key annealing temperatures. Briefly, $85 \%$ of the water is seen to dissociate first, with a corresponding growth of oxide upon heating to $400^{\circ} \mathrm{C}$. There is quantitative agreement between the oxygen available from the dissociated water $\left(2.5 \times 10^{\mathrm{95}} \mathrm{cm}-2\right)$ and the amount of additional oxide formed (Si-O bonds). Since the diffusion of water through silicon oxide is relatively facile at these temperature $\left(160 \AA \text { at } 150^{\circ} \mathrm{C}\right)^{27}$, these data are consistent with water diffusion and oxide formation on the silicon side of the interface. Note that, once most of the interfacial water is gone, the hydroxyl spectrum is shifted to $3740 \mathrm{~cm}^{-1}$, indicative of "free" (non H-bonded) hydroxyls, with a broad band centered at $3600 \mathrm{~cm}^{-1}$, characteristic of OH interacting with other $\mathrm{OH}$, not with water molecules. The interface between the two wafers is still well-defined, with absence of chemical bond between the surfaces.

The evolution of hydroxyl takes place between 400 and $800^{\circ} \mathrm{C}$, where the $\mathrm{O}-\mathrm{H}$ stretch band almost disappears while the oxide band increases. Interestingly, water molecules are reformed around $800^{\circ} \mathrm{C}$, suggesting that the hydroxyl dissociation mechanism is:

$$
-\mathrm{Si}_{\mathrm{i}}-\mathrm{OH}+-\mathrm{Si}_{\mathrm{i}}-\mathrm{OH} \Rightarrow \mathrm{Si}_{\mathrm{i}}-\mathrm{O}-\mathrm{Si}_{\mathrm{i}}+\mathrm{H}_{2} \mathrm{O}
$$

with subsequent diffusion/reaction of water to form oxide by $1000^{\circ} \mathrm{C}$. The subscript "i" in Eq. (3) denotes species at the internal surfaces of the two wafers.

Evidence for a chemical bond between the two surfaces can be seen in the oxide band, best shown in panel (b) of Fig. 11. The appearance of a shoulder above $1250 \mathrm{~cm}^{-1}$ has been correlated to the formation of strained $\mathrm{Si}-\mathrm{O}-\mathrm{Si}$ bridging linkages at the interface. Model calculations have shown that while isolated Si-O-Si quasi-linear species have a $\mathrm{Si}-\mathrm{O}$ stretch frequency at $1100 \mathrm{~cm}^{-1}$, a collection of $\mathrm{Si}-\mathrm{O}-\mathrm{Si}$ species sandwiched between two thin SiO2 layers is characterized by a collective LO phonon centered at $1265 \mathrm{~cm}^{-1}$ as observed. Incidently, the magnitude of this shoulder varies with position on the sample indicating that by $1000^{\circ} \mathrm{C}$, the chemical linkage between the two internal surfaces is still highly inhomogeneous. It is not surprising that the "bonding" temperature specified in manufacturing is $1100^{\circ} \mathrm{C}$, at which the formal interface has disappeared with no spectral evidence for $\mathrm{Si}-\mathrm{O}-\mathrm{Si}$ linkages (complete formation of $\mathrm{SiO}_{2}$ ).

Finally, the fate of hydrogen during these processes can be followed in panel (c) of Fig. 11. While $\mathrm{H}$ adsorbed directly on the Si surface $\left(2100 \mathrm{~cm}^{-1} \text { band }\right)^{28}$ undergoes transformations consistent with the rearrangement of the $\mathrm{Si}$ surface by $600^{\circ} \mathrm{C}$, the concentration of hydrogen inside the thin oxide $\left(2250 \mathrm{~cm}^{-1}\right.$ band $)^{28}$ increases as the hydroxyl groups react; upon $800^{\circ} \mathrm{C}$, the increase of the $2250-2300 \mathrm{~cm}^{-1}$ band suggests that the following reaction takes place, simultaneously with the water formation shown in Eq. (1):

$$
\mathrm{H}_{2} \mathrm{O} \rightarrow \mathrm{Si} / \mathrm{SiO}_{2} \text { interface } \Rightarrow \mathrm{SiO}_{2}+2 \mathrm{Si}-\mathrm{H}
$$

The hydrogen eventually diffuses away from the interface region, as seen in the spectrum at $1000^{\circ} \mathrm{C}$.

In summary, IR absorption spectroscopy gives a first look at the chemical bonding between to surfaces. Upon $1100^{\circ} \mathrm{C}$ anneal, hydrophobic surfaces have developed direct $\mathrm{Si}-\mathrm{Si}$ bonds and all the hydrogen has diffused away from the interface, while hydrophilic surfaces have their oxide chemically fused, after dissociation/reaction of water and hydroxyl groups and diffusion of hydrogen. This information gives a microscopic understanding of macroscopic bond strength measurements, and a better insight into process parameters for optimization of the bonding. 
a)

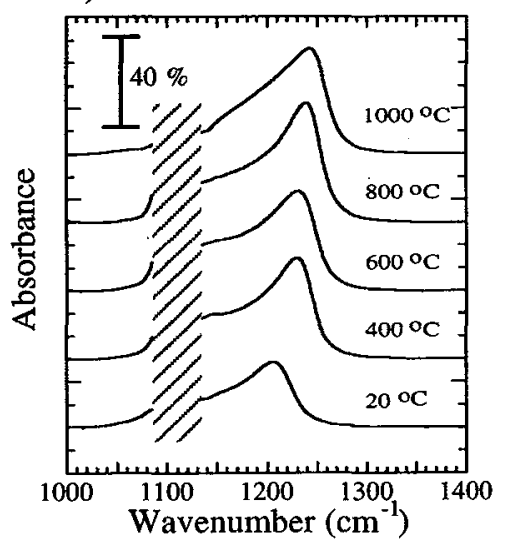

c)

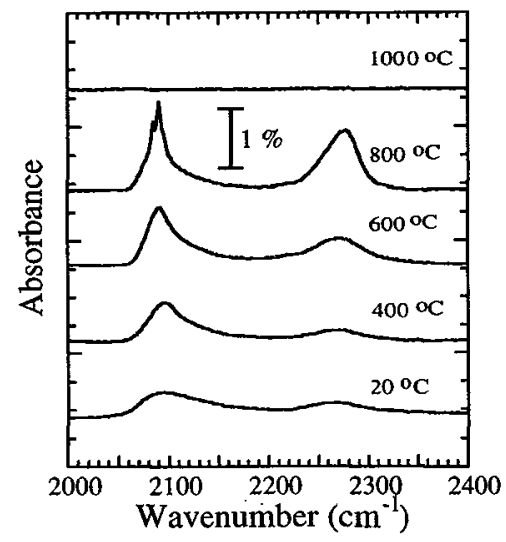

b)

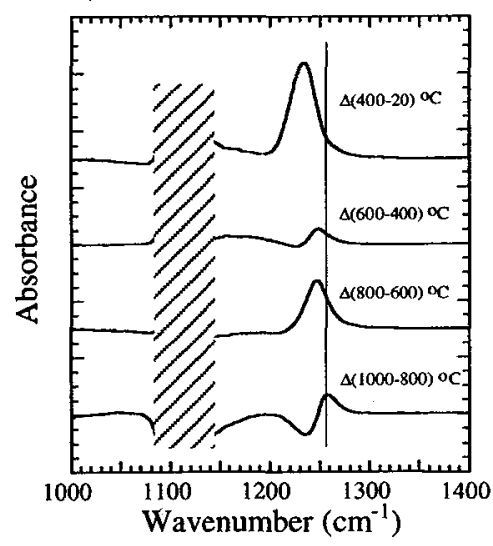

d)

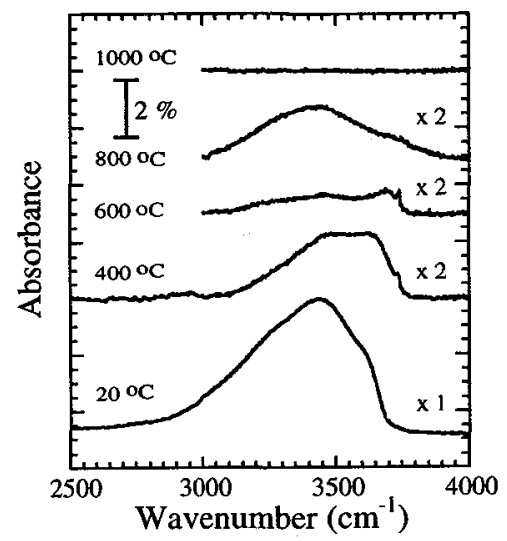

Figure 11. MIT spectra of the ratio of p:s polarization for the different spectral regions of interest for hydrophilic $\mathrm{Si}(111)$ as a function of annealing temperature: (a) Si-O phonon modes (b) Difference spectra $\left(\mathrm{T}_{2}-\mathrm{T}_{1}\right)$ derived from the Si-O spectra shown in (a). The solid vertical line indicates the lower bound of the absorbance attributed to formation of bridging $\mathrm{Si}-\mathrm{O}-\mathrm{Si}$ groups; the hashed area indicates the spectral region for which no IR throughput is observed, due to absorption by the interstitial oxygen present in these $\mathrm{CZ}$ samples; (c) $\mathrm{Si}-\mathrm{H}$ stretching modes; and (d) O-H stretching modes.

\subsection{Shearing mechanism}

The shearing mechanism upon $\mathrm{H}+$ implantation is particularly intriguing in view of the quality of SOI that car be obtained: control from $500 \AA$ to tens of microns with $+-50 \AA$ thickness variation! At each stage of the Controlled Wafer Shearing (CWS) process, IR spectroscopy can be used to monitor the chemical state of the 
implanted hydrogen and its evolution. The CWS process begins by the implantation of hydrogen $\left(10^{16}\right.$ to $10^{1 /}$ $\mathrm{H} / \mathrm{cm}^{2}$ ) through the surface of a wafer covered with an oxide layer (fig. 11a). Depending on the initial energy (typically $30 \mathrm{keV}$ to $1 \mathrm{MeV}$ ), the atoms will stop at an average depth ranging from about $4000 \AA$ to $16 \mu \mathrm{m}$, with a straggle varying from a few $100 \AA$ to about $1 \mu \mathrm{m}$. The implantation creates substantial damage in the silicon lattice, with a defect profile concentration similar to that of the hydrogen. IR spectroscopy has been used to demonstrate that most of the implanted hydrogen is initially trapped at point defects, and that agglomeration of these defects occurs to form $\mathrm{SiH}_{x(x=2,3)}$ species, upon annealing. We have recently extended these studies, to investigate the effect of joining the $\mathrm{H}$-implanted wafer to a support or handle wafer (fig.11) as discussed above. Specifically, we have used multiple internal reflection IR spectroscopy to demonstrate that extended defects, comprised of internal $\mathrm{H}$-terminated surfaces form at elevated temperatures, for doses above $6 \times 10^{16} \mathrm{H} / \mathrm{cm}^{2}$. These defects eventually evolve to become macroscopic cracks that subsequently cause the shearing of the wafer at the implanted layer, thus transferring the upper silicon to the handle wafer. Once again, IR spectroscopy has proven to be an immensely powerful for probing regions/layers inaccessible to other techniques.

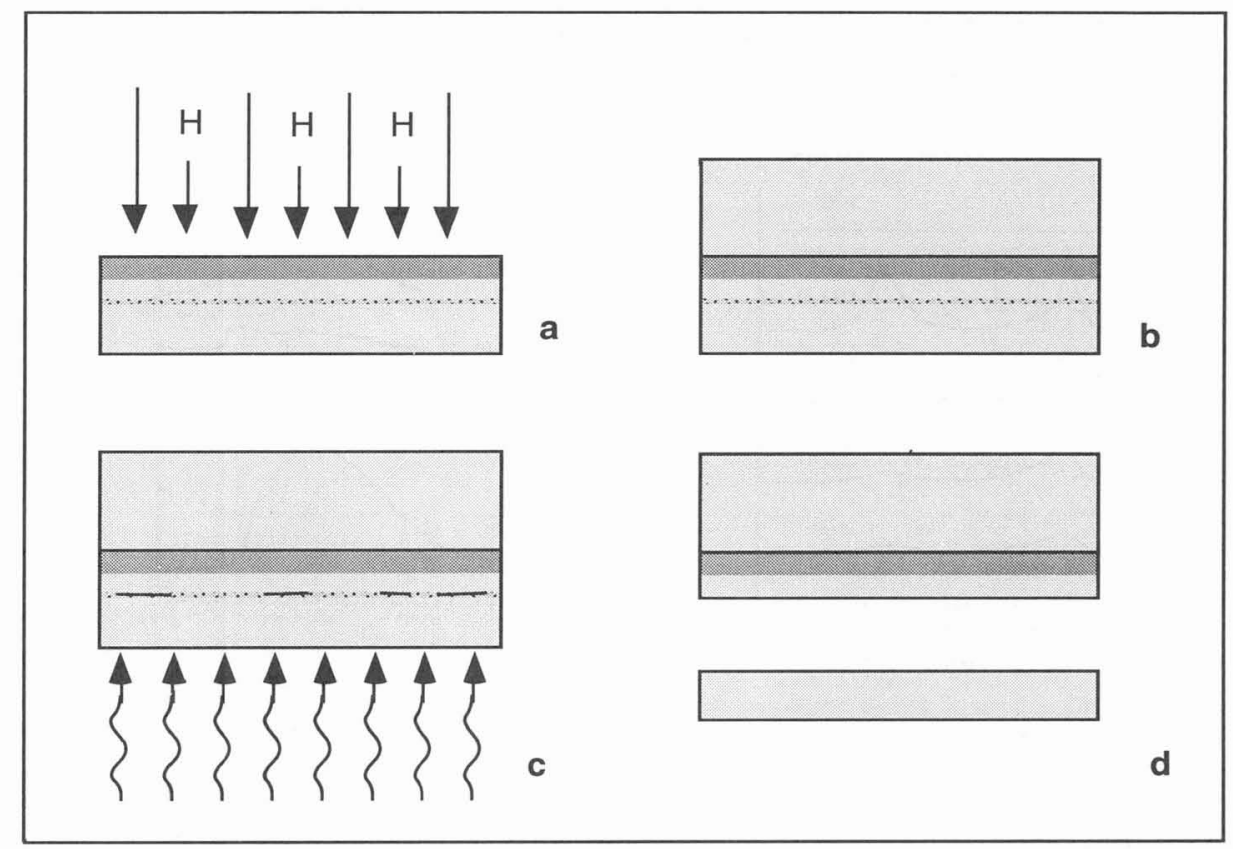

Figure 12. Principle steps involved in the CWS technique; a) implantation; b) wafer bonding; c) annealing and the final state with the creation of an SOI structure by transfer of a layer of silicon to the support wafer.

\section{CONCLUSIONS}

In this paper, we have summarized how infrared absorption spectroscopy can play a pivotal role in the evolution of microelectronics, not only by a powerful characterization of the surface and interfaces after different chemical treatments, but also in the development of a microscopic understanding of the novel phenomena that are associated with the fabrication of new materials. Despite this importance, relatively few fundamental studies of the physics and chemistry of semiconductor surfaces and interfaces appear in the surface science each year. It is therefore our hope that this review of our recent work may stimulate further experimental and theoretical studies within the surface science community by emphasizing the continuing need for such fundamental studies of these unique, technologically important systems. 


\section{References}

[1] Y.J. Chabal, in Handbook on Semiconductors, Vol. 2 "Optical Properties of Semiconductors", T.S. Moss and M. Balkanski, Eds. (Elsevier, Amsterdam, 1994) pp.187-234.

[2] Y.J. Chabal, M.A. Hines and D. Feijoo, J. Vac. Sci. Technol. A 13 (1995) 1719.

[3] G.S. Higashi and Y.J. Chabal, in Handbook of Silicon Wafer Cleaning Technology: Science, Technology and Applications, W. Kern Ed. (Noyes, Park Ridge, NJ, 1993) pp.433-496.

[4] W.G. Maszara, J. Electrochem. Soc. 138 (1991) 341.

[5] Proc. 1st, 2nd and 3rd Int'll Symp. on Semiconductor Wafer Bonding: Science,Technology and Applications, The Electrochemical Society Proceedings Series (Pennington, NJ, 1992-1995).

[6] A.J. Auberton-Herve, J.M. Lamure, T. Barge, M. Bruel, B. Aspar and J.L. Pelloie, Silicon-on-Insulator Technology (Semi-Con West, 1995).

[7] M.K. Weldon, Y.J. Chabal, D.R. Hamann, S.B. Christman, E.E. Chaban and L.C. Feldman, J. Vac. Sci. Technol. B14 (1996) 3095.

[8] J.D.E. McIntyre and D.E. Aspnes, Surf. Sci. 24 (1971) 417.

[9] W. Kern, Handbook of Silicon Wafer Cleaning Technology: Science, Technology and Applications (Noyes, Park Ridge, 1993).

[10] K. Kern, Semicond. Int. 94 (1984) April issue.

[11] E. Yablonovitch, D.L. Allara, C.C. Chang, T. Gmitter and T.B. Bright, Phys. Rev. Lett. 57, 249 (1986).

[12] M. Grundner and R. Schultz, in "Deposition and Growth Limits for Microelectronics", G.W. Rubloff and G. Lucovsky Eds., AIP Conf. Proc. 167 (1988) pp. 329-337.

[13] Y.J. Chabal, G.S. Higashi, K. Raghavachari and V.A. Burrows, J. Vac. Sci. Technol. A 7 (1989) 2104.

[14] G.S. Higashi, Y.J. Chabal, G.W. Trucks and K. Raghavachari, Appl. Phys. Lett. 56 (1990) 656.

[15] G.S. Higashi, R.S. Becker, Y.J. Chabal and A.J. Becker, Appl. Phys. Lett. 58 (1991) 1656.

[16] G.W. Trucks, K. Raghavachari, G.S. Higashi and Y.J. Chabal, Phys. Rev. Lett. 65 (1990) 504.

[17] R.J. Collins and H.Y. Fan; Phys. Rev. 93 (1954) 674.

[18] C.H. Bjorkman, T. Yamazaki, S. Miyazaki and M. Hirose, Proc. Int. Conf. on Advanced Microel. Dev. and Processing (1994) p.431.

[19] L.M. Struck, J. Eng, B.E. Bent, Y.J. Chabal, G.P. Williams, A.E. White, S.B. Christman, E.E.Chaban, K. Raghavachari, G.W. Flynn, K. Radermacher and S. Mantl, Mat. Res. Soc. Symp. Proc. 386 (1995) 395.

[20] Proc. of 1995 IEEE Int. SOI Conf. (Oct. 1995, Tucson, AZ).

[21] R. Stengl, K.-Y. Ahn and U. Gosele, Jpn. J. Appl. Phys. 27 (1988) L2364.

[22] M. Suhren, Y.J. Chabal, et al. (in preparation); M.K. Weldon and Y.J. Chabal (in preparation).

[23] M.K. Weldon, V.E. Marsico, Y.J. Chabal, D.R. Hamann, S.B. Christman and E.E. Chaban, Surf. Sci. xx (1996) xxx (Proceedings of the 8th Int'l conf. on Vibrations at Surfaces, in press).

[24] P. Hohenberg and W. Kohn, Phys. Rev. B 136 (1964) 864; W. Kohn and L.J. Sham, Phys. Rev. B140 (1965) A1133.

[25] D.R. Hamann, M. Schluter and C. Chiang, Phys. Rev. Lett. 43 (1979) 1494; D.R. Hamann, Phys. Rev. B 40 (1989) 2980; L. Kleinman and D.M. Bylander, Phys. Rev. Lett. 48 (1982) 1425.

[26] D. Feijoo, Y.J. Chabal, and S.B. Christman, Appl. Phys. Lett. 65 (1994) 2548.

[27] S.M. Sze, VLSI Technology (Mc Graw Hill, New York, 1983), Table 4 (Chapter 5, section 7).

[28] H. Ogawa and T. Hattori, Appl. Phys. Lett. 61 (1992) 577. 